\title{
Construcción colectiva de 'actitud cooperativa' desde un escenario virtual de aprendizaje
}

\author{
Carla Yeneris Caballero ${ }^{1}$ \\ Herman Van de Velde ${ }^{2}$
}

\section{RESUMEN}

Artículo basado en los resultados de una investigación que la autora realizó con el objetivo de aportar a la comprensión de cómo se puede construir colectivamente una actitud cooperativa desde escenarios virtuales de aprendizaje. El estudio constata que las estrategias metodológicas, los temas y un ambiente de afectividad fomentan una actitud cooperativa y permiten generar cambios sustanciales en el nivel de conciencia y los significados positivos que las/os participantes asignan a una experiencia educativa construida desde un enfoque de cooperación (genuina). La contribución teórica del estudio consiste en los sentidos y el concepto de cooperación genuina (5 pilares y 2 ambientes) vinculado a la construcción de una actitud cooperativa. Esta actitud integra 15 dimensiones y se relaciona con, al menos, 5 ejes del aprendizaje cooperativo. El aporte práctico son técnicas cooperativas que se pueden implementar desde un escenario virtual de aprendizaje. Las novedades se relacionan con el uso de plataformas educativas como mediadoras de procesos educativos, la incorporación de técnicas didácticas-tecnológicas adaptadas a lo virtual y la participación de una diversidad de personas en cursos virtuales de ABACOenRed y que comparten su visión pedagógica.

Palabras clave: cooperación genuina, actitud cooperativa, escenarios virtuales de aprendizaje

Recibido: 12 de septiembre de 2014

Aceptado: 10 de marzo de 2015

1 Egresada de la Maestría en Gestión del Desarrollo Comunitario, modalidad virtual. Facilitadora de procesos de aprendizajes (modalidad virtual y presencial). Experiencia como consultora de proyectos en aspectos relacionados a evaluaciones, sistematizaciones e investigaciones participativas..Correo Electroico: carla@abacoenred.com

2 Doctor en Ciencias Pedagógicas, docente UNAN-Managua / FAREM-Estelí y coordinador de ABACOenRed (www.abacoenred.com), un referente nuestroamericano como propuesta pedagógico-metodológica en construcción. Correo Electrónico: herman@abacoenred.com. 


\title{
Collective construction of 'cooperative attitude' from a virtual learning scenario
}

\begin{abstract}
Article based on the results of an investigation that the author conducted with the objective of contributing to the understanding of how to collectively build a cooperative attitude from virtual learning scenarios. The study noted that the approaches, themes and an atmosphere of affection foster a cooperative attitude and can generate substantial changes in the level of awareness and positive meanings that participants assign to an educational experience built from a cooperative approach (genuine). The theoretical contribution of the study consists of the senses and the concept of genuine cooperation (5 Pillars and 2 rooms) linked to the construction of a cooperative attitude. This approach integrates 15 dimensions and is related to at least 5 axis of cooperative learning. The practical contribution is cooperative techniques that can be deployed from a virtual learning scenario. The novelties relate to the use of educational platforms as mediators of educational processes, incorporating resource-technological techniques adapted to the virtual and the participation of a variety of people in virtual courses ABACOenRed and that share their pedagogical vision.
\end{abstract}

Keywords: genuine cooperation, cooperative attitude, virtual learning scenarios 


\section{INTRODUCCIÓN}

En la era tecnológica actual, cada vez más, los procesos educativos están siendo facilitados a través de plataformas, entornos o escenarios virtuales. Estas plataformas se han venido popularizando y constituyen una alternativa para quienes desean participar en procesos de aprendizajes con flexibilidad en la planeación de los tiempos de estudio; optimizando recursos y aprovechando herramientas pedagógicas adaptadas a tiempos modernos obviando las distancias físicas entre quienes participan en el proceso.

Por supuesto, un entorno virtual de aprendizaje, además de estar construido sobre una plataforma tecnológica, debe responder a una perspectiva pedagógicometodológica que facilite procesos de interacción social de cara a la formación virtual, en este caso de tipo cooperativo.

La cooperación integrada a los procesos educativos implica lo ya planteado por Pujolás (2009, p. 226), parafraseando a Slavin (1985), que es necesario "cooperar para aprender, para aprender a cooperar". Ovejero (1993) lo plantea también como una necesidad creciente para "evitar contextos educativos individualistas y competitivos que están profundizando la crisis de socialización y más bien se debe trabajar en contextos de interacción social cooperativa" (p. 379). Se trata, pues, de dar preeminencia a los procesos educativos que destaquen la colectividad y la construcción de oportunidades de aprendizaje para todas y todos, rescatando la cooperación desde un sentido didáctico, conceptual y cultural.

Es importante trabajar la cooperación como opción, posición y acción pedagógica considerando la construcción de una cultura cooperativa genuina, es decir cooperación genuina, que implica no solo un sentido didáctico y conceptual, sino también como enfoque cultural. Esto último nos posiciona en una situación contra hegemónica necesaria de construcción social y pedagógica.
Según Van de Velde (2013) el término 'cooperación genuina' se concibe como un acto educativo y una acción político-pedagógica que se basa en cinco pilares fundamentales desde su interrelación e integración creativa:

1. El arte de escuchar, llamada $A P E R T U R A$, que implica un abrirse a la escucha, estar atenta/o a lo que dicen las/os demás y también el contexto.

2. La habilidad de interpretar, LECTURA, lo que se ha escuchado decir a las/os demás con una actitud de profundo respeto a la diversidad particular según el contexto.

3. La voluntad de compartir, LA TERNURA, como la expresión máxima de solidaridad. Querer cooperar, querer compartir, ser solidaria/o con las/ os demás con respeto, desde el diálogo, desde la interculturalidad y con una actitud de servicio.

4. La decisión de compromiso, POSTURA, es decir, tomar una posición, expresarla y defenderla.

5. La visión de integración, LA CONTEXTURA, como una consideración a la globalidad en la que vivimos, al contexto global sin perder de vista lo local.

Estos 5 pilares deben entrelazarse a través de un ambiente de profunda CONFIANZA y capacidades de (AUTO) CRITICA CONSTRUCTIVA.

Pero... ¿Será que esto de cooperar es algo nuevo? ¿Integrar la cooperación en la educación es una novedad? Ni mucho menos, como nos dice Suárez (2008): "la escuela no requiere inventar la cooperación, lo que necesita es asumirla - e implicarla en los modelos pedagógicos- como condición social de aprendizaje" (p. 15). Tal como Suárez propone, se debe ser consciente que la cooperación, como estrategia de desarrollo humano, no requiere mucha presentación, es tan antigua como la humanidad que la ha validado en innumerables ocasiones para subsistir, progresar y sobre todo, configurar lo propiamente humano con otras/os, la cultura. 
Efectivamente, no es que la cooperación requiera mucha presentación, sino más bien, que los modelos sociales y, por ende los modelos educativos, están inspirados en una visión del "mundo mercado" que sostiene el paradigma del "desarrollo" que se expresa en las acumulaciones desproporcionadas y las exclusiones, la riqueza exagerada y la pobreza alarmante.

¿Cuál debería ser entonces la propuesta? Debería ser desarrollar procesos educativos basados en la cooperación, dejarse inspirar por otra visión del mundo, una visión contextual que se base en los valores de la solidaridad, la acción comunitaria, la convivencia armónica con la naturaleza y que persiga la construcción de comunidades sostenibles y felices. Esa propuesta alternativa del mundo rompe con el individualismo y debe ser construida mediante referentes éticospedagógicos y metodológicos alternativos que se conviertan en procesos de construcción colectiva de oportunidades para aprender, integrantes de una actitud cooperativa emprendedora de calidad creciente.

Al hablar de 'actitud cooperativa' nos referimos a un 'estado complejo de integración, relativamente estable, de 15 dimensiones, relacionado con los 5 pilares y 2 ambientes de la cooperación genuina'. Se nombra como 'actitud cooperativa' para enfatizar que no solo se considera a los tres componentes comúnmente propuestos por la psicología social (cognitiva, afectiva y conductual), sino que se retoma de la propuesta de ABACOenRed 12 componentes más (nombrándolos más bien como dimensiones ${ }^{1}$ ).

A continuación las 15 dimensiones de la actitud cooperativa que son retomados en el instrumento que se desarrolló en esta investigación (la escala de actitud) para valorar la actitud cooperativa:

1. Dimensión cognitiva, relacionada con
los conocimientos teórico-prácticos, las

1 Se prefiere el uso de la palabra 'dimensión' por ser un término que denota una visión holística e integradora de los fenómenos vitales. Dimensión refiere a un mayor alcance, magnitud e integralidad, en donde pueden caber uno o varios componentes. interpretaciones cognitivas, creencias.

2. Dimensión afectiva, que se expresa en las experiencias vividas, interpretaciones sentidas y emociones.

3. Dimensión conductual, que son las formas expresas de relacionarse activamente con su ambiente, tanto social, natural y material.

4. Dimensión político-ideológica que se refleja en expresiones de compromiso desde las posiciones políticas e ideológicas que cada persona tiene.

5. Dimensión económica, que implica la relación de las personas con los medios de producción.

6. Dimensión ética, que se expresa en los valores y las normas que se comparten como todo un proceso de negociación y se constituyen en un sistema propio.

7. Dimensión histórico-cultural que permite una mayor comprensión de por qué las personas somos quienes somos y cómo nos expresamos.

8. Dimensión cívica que indica la relación de fondo que la persona establece con el estado y abarca su posición ciudadana. Integra su sentido de responsabilidad social.

9. Dimensión estética, referida a la capacidad del disfrute, de la contemplación viva, tanto de lo que una/o misma/o hace como de lo que hacen otras/ os, tanto de su propio ser, como de los seres otros y del ser comunitario, de nuestro ser como colectivo, como sociedad.

10. Dimensión bio-energética, referida al estado del cuerpo humano, el ambiente y la salud tanto física como psico-social.

11. Dimensión volitiva, que se refleja en la voluntad, deseo o capacidad de decidir.

12. Dimensión psicomotora, que incorpora el conjunto de hábitos, capacidades, habilidades, aptitudes y destrezas necesario para el ejercicio con calidad de una determinada tarea.

13. Dimensión lúdico-artístico-creativa, relacionada directamente con la capacidad humana de expresarse y de crear.

14. Dimensión social o inter-relacional, la cual hace referencia al 'estado de salud' de nuestras relaciones 
tanto en lo material como en lo social.

15. Dimensión biológica que refleja, dentro de toda actitud, la relación intrínseca del ser humano con la naturaleza (medio ambiente).

Todo lo anterior es una 'esquematización', o más bien 'estructuración' del fenómeno 'actitud' y se debe integrar un enfoque holístico que implica que toda actitud en la vida es única, integral, integradora e integrada. Sirve esta estructuración para el análisis teórico, sin embargo, también puede tener una 'utilidad práctica' bastante accesible, a pesar de que resulte aparentemente contradictorio con el enfoque holístico: si en una determinada situación, se logra 'descomponer' la actitud y visualizar, tanto componentes fuertes, como débiles, entonces se podrían identificar posibles pistas por dónde entrar para lograr un aprendizaje (cambio de actitud) de mayor calidad.

Con el objetivo de aportar a la comprensión de cómo se puede construir colectivamente una actitud cooperativa en escenarios virtuales de aprendizaje, y reconociendo la complejidad del fenómeno de la construcción de una cultura de cooperación en una experiencia de formación virtual, es que se realizó la investigación de la que se deriva el presente artículo.

El objeto del estudio fue la comprensión del nivel de actitud cooperativa de las/os participantes en cursos virtuales, facilitados concretamente desde ABACOenRed, desde su plataforma educativa virtual y coherente con su referente ético-pedagógicometodológico.

En el estudio se identificaron los aspectos que inciden en la construcción de esta actitud y partiendo del supuesto inicial que uno de estos elementos es la incorporación de técnicas grupales o cooperativas en el proceso de aprendizaje.

\section{MATERIALES Y MÉTODOS}

Herramientas metodológicas esenciales de planeación del proceso de investigación fueron la visión vertical y la visión horizontal.

En la visión vertical se incluyó un acercamiento a la razón de ser del proceso investigativo, la situación problémica/sueño, problema científico y sus preguntas orientadoras, objeto y sujetos de estudio, campo de acción, sistema de objetivos y tipo de investigación, así como una bibliografía básica inicial.

La visión horizontal presenta en forma de matriz, las principales interrogantes científicas del estudio y sus correspondientes tareas, métodos, resultados y 'posible salida' en la estructura final de la tesis.

La ruta metodológica del proceso de investigación se dividió en 6 momentos metodológicos generales, desde la preparación del proceso de investigación, la definición y preparación de instrumentos metodológicos, el trabajo de campo hasta el procesamiento, análisis e interpretación de datos para elaborar el informe final que correspondió directamente al sistema de objetivos planteados.

Con respecto a los métodos, a nivel teórico se aplicaron:

- La revisión documental y su correspondiente análisis de contenido para la construcción del referente teórico-conceptual-experiencial. Vale mencionar que como preámbulo de la investigación se abrió un foro en LinkedIn ${ }^{2}$ para crear un debate y análisis entre distintos profesionales latinoamericanos acerca de sus concepciones sobre los conceptos cooperar-colaborar y cómo inciden las técnicas cooperativas para fomentar una cultura de cooperación a lo interno del grupo de participantes en procesos virtuales. Estas reflexiones sirvieron para nutrir el referente teórico-experiencial y afinar las ideas iniciales del proceso de investigación.

2 Red Social orientada a establecer relaciones profesionales e 45 
Estimada/o amiga/0:

Te invitamos a que compartas tu valoración sobre las siguientes frases, las cuales están relacionadas con distintas preferencias en cuanto a una variedad de formas de trabajo. Tenemos respeto profundo por la diversidad de ideas y prácticas reconociendo la complementariedad necesaria que garantiza la calidad del trabajo en cada contexto.

Antes, nos gustaría saber algunos de tus datos personales:

Sexo: Mujer $\bigcirc$ Hombre $\bigcirc$ Edad: Profesión: Función laboral (actual):

Marca con una $\mathrm{X}$ en la columna que corresponda de acuerdo a la escala planteada.

\begin{tabular}{|c|c|c|c|c|c|c|c|c|c|}
\hline \multirow[t]{2}{*}{$\#$} & \multirow[t]{2}{*}{ Escala: } & \begin{tabular}{|l|l|}
1 & Definitivamente, NO es asi (totalmente en desacuerdo) \\
2 & Considero que NO es asi \\
3 & Me parrece que NO es asi \\
4 & Realmente NO SE si será asi (zi de asuetds, zi en ectacerta) \\
5 & Me parece que asi es \\
\end{tabular} & \multicolumn{7}{|c|}{$\begin{array}{l}\text { OJO: Revisa muy bien lo que } \\
\text { significa cada número (ver } \\
\text { escala al lado izquierdo) }\end{array}$} \\
\hline & & \begin{tabular}{|l|l|}
6 & Considero que si, asi es \\
7 & Definitivamente, asi es (totalmente de acuerdo) \\
\end{tabular} & 1 & 2 & 3 & 4 & 5 & 6 & 7 \\
\hline 01 & Sólo trabajo & a equipo cuando me lo orientan explícitamente. & & & & & & & \\
\hline 02 & Al trabajar er & equipo resuelvo mejor las tareas. & & & & & & & \\
\hline 03 & En el trabajo & n equipo, uno/a somos todos/as y todos/as somos uno/a. & & & & & & & \\
\hline 04 & Si trabajo sol & 0 , soy más eficiente. & & & & & & & \\
\hline 05 & $\begin{array}{l}\text { No hago nad } \\
\text { nada. }\end{array}$ & a favor del medio ambiente, porque sólo mi esfuerzo no sirve de & & & & & & & \\
\hline 06 & Cuando traba & o en equipo, me estanco y no desarrollo plenamente mis talentos. & & & & & & & \\
\hline 07 & $\begin{array}{l}\text { Mientras más } \\
\text { acuerdo. }\end{array}$ & opiniones hay sobre un trabajo, nos cuesta más ponernos de & & & & & & & \\
\hline 08 & Desde mi pur & o de vista, cooperar y colaborar es lo mismo. & & & & & & & \\
\hline 09 & $\begin{array}{l}\text { De nada sirve } \\
\text { cuenta nuestr }\end{array}$ & $\begin{array}{l}\text { que trabajemos en equipo porque, con frecuencia, no tomamos en } \\
\text { s opiniones. }\end{array}$ & & & & & & & \\
\hline 10 & Nuestra sobr & iivencia está determinada por nuestra capacidad de cooperación. & & & & & & & \\
\hline 11 & El trabajo en & quipo nos permite ir construyendo un ambiente de confianza. & & & & & & & \\
\hline 12 & Si trabajamos & en equipo, compartimos metas. & & & & & & & \\
\hline 13 & Reunirme en & quipo me gusta, aunque me sienta mal de salud. & & & & & & & \\
\hline 14 & Soy más crea & ivo/a cuando trabajo solo/a. & & & & & & & \\
\hline 15 & Al poder eleg & r entre trabajar en equipo y trabajar sola/o, prefiero hacerlo solo/a. & & & & & & & \\
\hline 16 & Si compito, a & canzo mejor mis metas. & & & & & & & \\
\hline 17 & El trabajo en & quipo me permite desarrollar más mis habilidades. & & & & & & & \\
\hline 18 & $\begin{array}{l}\text { Mi relación c } \\
\text { de clase no in }\end{array}$ & $\begin{array}{l}\text { n los miembros de mi familia, mi comunidad y mis compañeros/as } \\
\text { luyen mucho en mi persona. }\end{array}$ & & & & & & & \\
\hline 19 & $\begin{array}{l}\text { Cuando traba } \\
\text { sentimientos. }\end{array}$ & amos en equipo, respetamos la diversidad de opiniones y & & & & & & & \\
\hline 20 & $\begin{array}{l}\text { La forma en } \\
\text { en un equipo }\end{array}$ & $\begin{array}{l}\text { ue me relaciono con otras personas, se refleja en cómo me integro } \\
\text { le trabajo. }\end{array}$ & & & & & & & \\
\hline 21 & Con buena sa & ud fisico-mental trabajo mejor individualmente. & & & & & & & \\
\hline 22 & $\begin{array}{l}\text { Al compartir } \\
\text { crear ideas su }\end{array}$ & $\begin{array}{l}\text { nis ideas en un equipo, éstas se enriquecen, lo que me permite } \\
\text { veriores. }\end{array}$ & & & & & & & \\
\hline 23 & $\begin{array}{l}\text { Saber interpr } \\
\text { común. }\end{array}$ & tar nuestros aportes en equipo es importante para cumplir una meta & & & & & & & \\
\hline 24 & Para que nue & ro pais salga adelante, debemos trabajar juntas/os. & & & & & & & \\
\hline 25 & Mi comunida & y yo, constituimos una unidad inseparable. & & & & & & & \\
\hline 26 & $\begin{array}{l}\text { Con el trabaj } \\
\text { tarea. }\end{array}$ & individual resuelvo más rápido y con mejor calidad cualquier & & & & & & & \\
\hline 27 & $\begin{array}{l}\text { Tengo que te } \\
\text { equipo con } \mathrm{c}\end{array}$ & $\begin{array}{l}\text { er voluntad de compartir como condición, para un trabajo de } \\
\text { idad. }\end{array}$ & & & & & & & \\
\hline 28 & En los equipo & de trabajo, muchas veces no nos sabemos escuchar. & & & & & & & \\
\hline 29 & Trabajar ene & uipo es una expresión cultural nuestra. & & & & & & & \\
\hline 30 & Me siento $\mathrm{me}$ & cómodo/a trabajando solo/a porque así no me critican tanto. & & & & & & & \\
\hline
\end{tabular}

- El enfoque sistémico utilizado en la interpretación de las relaciones entre los datos obtenidos por la vía empírica en el proceso de valoración de la construcción de una actitud cooperativa y los factores incidentes en un escenario educativo virtual.

- El método vivencial que permitió la incorporación de la reflexión teórica desde la experiencia de la autora de la tesis como facilitadora de procesos de aprendizajes en escenarios educativos virtuales, así como las expresiones subjetivas de las/os participantes expresadas a través de los métodos empíricos.

- También se aplicó el análisis, síntesis einducción comométodos que permitieron extraer de los datos la información resultante del proceso investigativo.

\section{Los métodos a nivel empírico:}

- La escala tipo Likert la cual permitió valorar, de forma eminentemente cualitativa y subjetiva, la actitud de las/os miembros de la comunidad con respecto a la cooperación y en el trabajo en equipo (ver figura \#1).

- Los conversatorios abiertos utilizando medios virtuales (chat por Skype o Facebook y los intercambios en los foros y chat).

- Los grupos o chat focales que, a través de preguntas guía, permitieron conocer los sentires y pensares de las/os participantes en cuanto a la construcción de la actitud cooperativa (se llaman chat focales porque fueron realizados por medio de la sala de chat de la plataforma y por desarrollar un tema 'focal' de reflexión).

\section{- Entrevista escrita basada en} una guía de evaluación con las que se pretendió integrar diferentes puntos de vista respecto a la valoración del proceso desarrollado: la auto-evaluación crítica, la co-evaluación entre participantes y particularmente en el grupo-base, así como la evaluación general del diplomado, incluyendo también el rol de facilitación. En esta guía se incluyeron escalas de valoración de 1 a 10 en donde 1 era totalmente insatisfecha/o y $10-$ totalmente satisfecha/o. A la guía de evaluación se integró un instrumento especial de auto-percepción (ver figura \#2) para que cada una/o de las/os 
participantes valorara su actitud cooperativa a partir del ejercicio de los 5 pilares y los 2 ambientes de la 'Cooperación Genuina'.

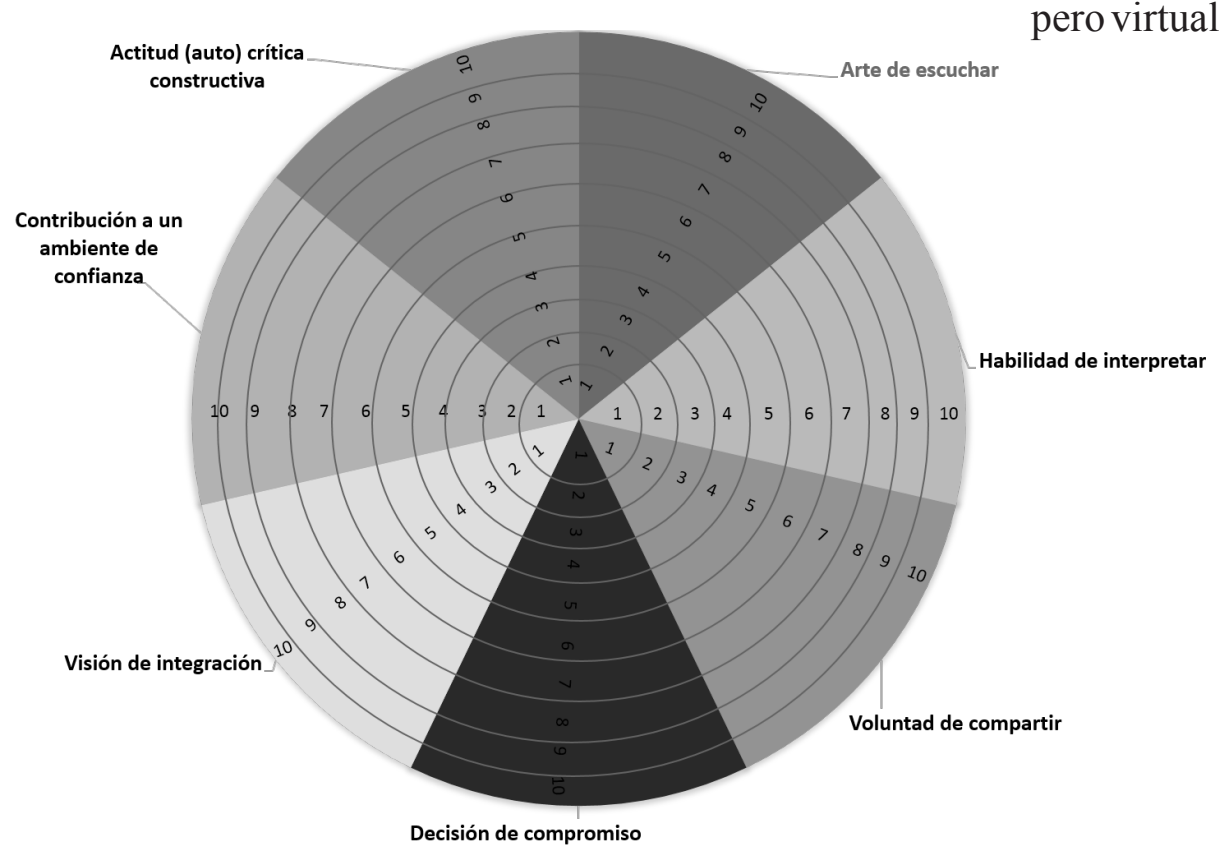

virtual de Moodle.

- La observación participante la cual brindó la posibilidad inmersiva, consciente y sistemática, pero virtual, de compartir las actividades, intereses y afectos desde dentro, como compañera más de aprendizaje sin delatar la condición de investigadora (Suárez, 2010), para poder explicar desde fuera la dinámica de construcción de una actitud cooperativa.

La investigación, eminentemente cualitativa, se basó en el método descriptivo y utilizó un proceso inductivo tratando de desarrollar conceptos, interacciones y comprensiones partiendo de pautas de los datos emergentes.
- Escritos o ensayos personales sobre la valoración general del diplomado enfatizando en la construcción de la actitud cooperativa y sus lecciones aprendidas en el proceso.

- La triangulación como una forma de constatar los resultados obtenidos, a través de los distintos métodos empíricos, que posibilitó integrar la información recogida sobre cómo dicen que interactúan o construyen actitud cooperativa (escala Likert, entrevistas, guías de evaluación, escritos, conversatorios), cómo es que interactúan (a través del análisis de los mensajes escritos en los foros y chat) y cómo se ha interactuado cooperativamente (a través de la observación participante) por medio de todas las herramientas tecnológicas del aula
La muestra elegida (invitada), o la comunidad de referencia del estudio, es la conformada por las/os participantes en el VIII Diplomado en Facilitación y Acompañamiento de Procesos de Aprendizajes $(\text { DI-FACIL } 8)^{3}$ de la cual la autora del artículo fue la facilitadora.

La muestra productiva, es decir, quienes respondieron debidamente a la escala de valoración (inicial y final), fueron 14 personas (10 mujeres y 4 hombres); en el caso de los conversatorios abiertos con informantes clave fueron 3, en los chat focales fueron 10; quienes respondieron a la guía de evaluación fueron 13 y quienes completaron debidamente el instrumento especial para valorar el avance en cuanto a los pilares/ambientes

\footnotetext{
3 Este diplomado fue creado específicamente en función de esta investigación y se realizó entre los meses de febrero y junio de 2014. Contó con la participación de 21 personas de 8 países: República Dominicana, Honduras, Ecuador, España, Chile, Colombia, México y Nicaragua. Este diplomado, además de definir el escenario tecnológico (o virtual) del trabajo de campo de esta investigación, es parte de los cursos que se desarrollan desde la plataforma educativa de ABACOenRed (www.abacoenred.com), tal que se logró un ambiente natural y muy parecido a otros cursos o diplomados desarrollados por ABACOenRed. El curso fue diseñado en Moodle en donde se desarrollaron actividades sincrónicas y asincrónicas.

El objetivo del diplomado es que en forma colectiva las/os participantes - incluyendo su facilitadora - logren identificarse (saber SER) con características de un aprendizaje cooperativo de calidad creciente, saber (comprender y hacer) cómo se construye y cómo se puede facilitar y valorar.
} 
de la cooperación genuina fueron 12 personas. Así que la muestra productiva varió dependiendo de los instrumentos de recogida de datos que se utilizaron.

\section{RESULTADOS Y DISCUSIÓN}

Gracias a la complementariedad de los distintos métodos teóricos y empíricos utilizados en el proceso investigativo, la investigación permitió estructurar los siguientes resultados:

La revisión documental y su correspondiente análisis de contenido permitieron fundamentar la 'esencia' en la que se basa la construcción de actitudes cooperativas en procesos de formación, desde una modalidad virtual de aprendizaje. En este proceso se determinó que la 'actitud cooperativa' es un estado complejo de integración, relativamente estable, de 15 dimensiones, relacionado con los 5 pilares y 2 ambientes de la cooperación genuina.

Lo anterior quiere decir que la 'actitud cooperativa' es inter-dimensional y tiene que ver con lo que 'cada una/o somos' (no solamente con las expresiones visibles de cómo actuamos, sino también qué y cómo nos sentimos, qué queremos, cómo nos proyectamos, cómo vivimos...) y que se desarrolla en un contexto socio-histórico-cultural en el que la confianza y la actitud (auto) crítica es fundamental.

Fundamentar la esencia de la construcción de actitudes cooperativas también permitió comprender que existen teorías psicopedagógicas y sociales (entre ellas: teoría genética de Piaget y su desarrollo a través de la Escuela de Psicología Social de Ginebra (1896-1980); teoría sociocultural de Vygotsky (18961934); teoría de la interdependencia social de los hermanos Johnson (1999); aprendizaje significativo de Ausubel (1918-2008); la Psicología Humanista de Rogers (1902-1987); la teoría de las inteligencias Múltiples de Gardner (2010) y la Pedagogía de SER de ABACOenRed (2004)) que sustentan ejes del aprendizaje cooperativo que las/os facilitadores/as de procesos pueden tomar en cuenta para orientar los esfuerzos educativos hacia el fortalecimiento de las actitudes cooperativas.

Para desarrollar una cultura de cooperación es esencial desarrollar estrategias metodológicas que incorporen técnicas grupales o colectivas y considerar la conformación de equipos o grupos-base de trabajo como unidades básicas de interacción social permanente.

En el proceso de análisis también se consideraron ejes fundamentales del aprendizaje cooperativo ${ }^{4}$. En el estudio se retoman los ejes sugeridos por Suárez (2010) integrando otros elementos de la propuesta pedagógicometodológica desarrollada por ABACOenRed: Gestión interna del equipo, evaluación interna del equipo, interdependencia positiva, interacción estimuladora y responsabilidad personal y compartida (de equipo).

A través de la aplicación de una escala de actitud se pudo determinar el punto de partida en cuanto a actitud cooperativa que tenían las/os participantes en la experiencia educativa que fue desarrollada a través de un escenario tecnológico virtual. Se constató que las/os participantes conceden mucha importancia a la interdependencia positiva (cooperativa) y al escenario en el cual se desarrolla la experiencia, el cual debe estar caracterizado por un ambiente de confianza y de respeto que les permita compartir sus opiniones/sentimientos y evitar una interacción de oposición (competir) para poder construir sinergia e identidad cooperativa. El aspecto que más les preocupó, inicialmente, fue el reconocimiento de la poca capacidad de escucha que caracteriza comúnmente a los integrantes de un grupo de trabajo y que puede incidir negativamente en los procesos cooperativos grupales.

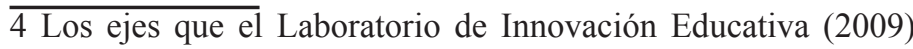
propone son: 1) agrupamientos heterogéneos; 2) Interdependencia positiva; 3) Responsabilidad personal; 4) Igualdad de oportunidades en el aprendizaje compartido; 5) Interacción promotora; 6) Procesamiento cognitivo de la información; 7) Utilización de habilidades cooperativas y 8) Evaluación grupal. 
Se desarrollaron y validaron 5 técnicas grupales o colectivas (cooperativas): convenio cooperativo, mapas o esquemas mentales colectivos, técnica de aprendizaje cooperativo cruzado en un ambiente virtual, producción creativa en cadena cooperativa y dúos o tríos de escritura cooperativa ${ }^{5}$. Estas técnicas fueron aplicadas a lo largo de la experiencia educativa virtual y reforzaron el sentido de responsabilidad personal y de equipo, la interdependencia positiva, la interacción promotora y la propia gestión interna de los grupos. Por supuesto, no solamente la estrategia metodológica (lo didáctico) aplicada reforzó los ejes antes mencionados, sino también las propias temáticas que hacían mucho énfasis en lo cooperativo (lo conceptual) y las relaciones de afectividad, especialmente el valor de la solidaridad que las/ os participantes compartieron entre todos/as los participantes y especialmente con los integrantes de su grupo-base (lo cultural).

A través de la aplicación de los instrumentos (escala de valoración final, guías de conversatorios abiertos, guías de evaluación de la experiencia con el instrumento especial para valorar los pilares/ambientes de la cooperación genuina, análisis de los ensayos personales y de los intercambios en foros y chat) se lograron recolectar datos que permiten rescatar las percepciones subjetivas y los significados que las/ os participantes asignaron a la experiencia educativa y la valoración del aporte de toda la experiencia (incluyendo la aplicación de técnicas cooperativas) en el desarrollo de su actitud cooperativa.

A través de la aplicación final de la escala de actitud se verificó un ligero aumento (de $\mathbf{0 . 1 4 3}$ ) en cuanto a los niveles de actitud cooperativa reportada al inicio y al final de la experiencia educativa. También se constata un progreso positivo en las dimensiones y los ítems (positivos y negativos) que conforman la escala de actitud.

5 Las técnicas grupales son inéditas y fueron desarrolladas por la autora junto con el equipo facilitador de ABACOenRed.
El análisis del contenido de los ítems permite constatar un cambio en las percepciones de las/os participantes con respecto a los factores que inciden en los procesos cooperativos. Si bien al inicio del diplomado (aplicación inicial de la escala) concedían mucha importancia a las condiciones que esperaban encontrar para desarrollar la experiencia (confianza, respeto, escucha, metas comunes...), al final del proceso valoran más los aspectos motivacionales y la razón de ser del trabajo en equipo, considerando los beneficios y los retos que supone.

Las guías de evaluación (auto-evaluación, coevaluación y valoración general del diplomado), los ensayos y la revisión de los intercambios en foros y chat permitieron extraer testimonios y valoraciones subjetivas muy positivas que las/os participantes tienen de la experiencia. Aunque se reconocen algunas limitantes surgidas en el proceso también se reconocen avances positivos y progresivos en cuanto a los ejes de la actitud cooperativa (responsabilidad personal y de equipo, la interdependencia positiva, la interacción promotora y la propia gestión interna de los grupos) especialmente en el hecho de haber podido compartir, dialogar, argumentar, interactuar y aprender nuevas metodologías, tecnologías y enfoques de trabajo, especialmente de lo cooperativo.

\section{Con el instrumento especial sobre los pilares/}

ambientes de la cooperación genuina y su relación con la construcción de una actitud cooperativa se logró constatar un aumento colectivo en el ejercicio de estos pilares/ambientes por el hecho de haber participado en la experiencia educativa (+1.55 de aumento). Si bien, esto es una expresión subjetiva, no deja de ser importante ya que todas/os las/os participantes, sin excepción, afirman que han aumentado su práctica en el ejercicio de cada pilar o ambiente. Se debe tener conciencia que la percepción subjetiva es parte esencial de la realidad que vive cada persona ya que al valorar personalmente que he avanzado, es un elemento motivador para seguir progresando en los aspectos que tienen un significado personal para cada ser humano. 


\section{CONCLUSIÓN Y RECOMENDACIONES}

De forma general se concluye que se logró constatar que las estrategias metodológicas que fomentan una actitud cooperativa permiten generar cambios sustanciales en el nivel de conciencia y los significados positivos que las/os participantes asignan a una experiencia educativa - desde un espacio virtual - construida desde un enfoque de cooperación (genuina). Por supuesto, estas estrategias metodológicas deben ser reforzadas por temáticas que hagan énfasis en lo cooperativo y promover, desde el inicio, un ambiente favorable que permita el desarrollo de relaciones de afectividad, especialmente el valor de la solidaridad.

La metodología, los temas y el ambiente son elementos esenciales de los 'escenarios educativos' que necesitan crearse para favorecer el desarrollo de una actitud cooperativa.

Se recomienda continuar elaborando instrumentos 'científicos' que nos acerquen a la comprensión de cómo se construye una actitud cooperativa, dimensiones, factores y todos sus elementos subyacentes. Seguir validando instrumentos aplicados para 'valorar' la actitud cooperativa, tanto la escala como el instrumento de auto-percepción.

También sería importante crear y documentar más técnicas cooperativas a desarrollar en contextos virtuales y que apunten al fortalecimiento de actitudes cooperativas de quienes participamos en procesos educativos.

\section{BIBLIOGRAFÍA}

García, M. y Cotrina, M. (2012). “Andamiando el desarrollo de los grados de magisterio: la cooperación educativa en el contexto universitario", REDU Revista de docencia universitaria, Vol. 10, 3, 399-414. España.

Laboratorio de Innovación Educativa (2009).
Aprendizaje cooperativo: propuesta para la implantación de una estructura de cooperación en el aula. España.

Ovejero Bernal, A. (1993). “Aprendizaje cooperativo: una eficaz aportación de la psicología social a la escuela del siglo XXI”, Psicothema, Vol 5, 373391. España:

Pujlás,P.(2008).Lacalidaddelosequipos de aprendizaje cooperativo: algunas consideraciones para el cálculo del grado de cooperatividad.

Suárez Guerreo, C. (2010). “Aprendizaje cooperativo e interacción asíncrona textual en contextos educativos virtuales", Medios y Educación, No. 36, 53-67. España.

Suárez Guerrero, C. (2008). Cooperación como condición social de aprendizaje. España: editorial UOC.

Suárez, C. (2003). "Del aprendizaje en red a una red de aprendizaje”, El Tintero, Vol. 3, 10, 1-7. México.

Van de Velde, H. (2010). Cooperar - jun acto esencialmente educativo!. Estelí: ABACOenRed.

Van de Velde, H. (2010). Evaluación de los efectos de la cooperación de la ONG belga 'VOLENS' en la Educación Alternativa de jóvenes excluidas/os de las laderas del Trópico Seco en Centroamérica (2008-2009), desde las organizaciones socias. Tesis en opción al grado de Doctor en Ciencias Pedagógicas. Instituto Pedagógico Latinoamericano y Caribeño (IPLAC), La Habana, Cuba.

Van de Velde, H. (2013). Cooperación genuina: un sueño a concretar que implica educación alternativa. Estelí: ABACOenRed. .

Van de Velde, H. (2013). La cooperación genuina, base para una Educación Alternativa, también a nivel de la Educación Superior. Estelí: UNAN Managua/FAREM Estelí.

Van de Velde, H. (2013). RETO para la Educación en Nicaragua 2013: construir calidad educativa, basada en cooperación genuina. Estelí: ABACOenRed. 